\title{
Homotypic dimerization of a maltose kinase for molecular scaffolding
}

SUBJECT AREAS:

MOLECULAR

CONFORMATION

X-RAY CRYSTALLOGRAPHY

Received

22 May 2014

Accepted

8 July 2014

Published

23 September 2014

Correspondence and requests for materials should be addressed to Z.R. (raozh@nankai. edu.cn)

* These authors contributed equally to this work.

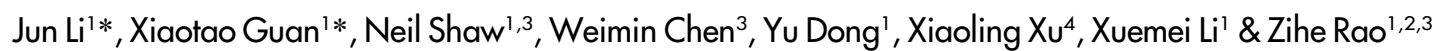

'National Laboratory of Biomacromolecules, Institute of Biophysics, Chinese Academy of Sciences, 15 Datun Road, Beijing, 100101, China, ${ }^{2}$ Structure Biology Laboratory, Tsinghua University, Beijing, 100084, China, ${ }^{3}$ Tianjin Key Laboratory of Protein Science, College of Life Sciences, Nankai University, Tianjin, 300071, China, ${ }^{4}$ Institute of Aging Research, School of Medicine, Hangzhou Normal University, 310036 , China.

Mycobacterium tuberculosis (Mtb) uses maltose-1-phosphate to synthesize $\alpha$-glucans that make up the major component of its outer capsular layer. Maltose kinase (MaK) catalyzes phosphorylation of maltose. The molecular basis for this phosphorylation is currently not understood. Here, we describe the first crystal structure of $M t b M a K$ refined to 2.4 A resolution. The bi-modular architecture of $M t b M a K$ reveals a remarkably unique $\mathrm{N}$-lobe. An extended sheet protrudes into ligand binding pocket of an adjacent monomer and contributes residues critical for kinase activity. Structure of the complex of MtbMaK bound with maltose reveals that maltose binds in a shallow cavity of the C-lobe. Structural constraints permit phosphorylation of $\alpha$-maltose only. Surprisingly, instead of a Gly-rich loop, MtbMaK employs 'EQS' loop to tether ATP. Notably, this loop is conserved across all MaK homologues. Structures of MtbMaK presented here unveil features that are markedly different from other kinases and support the scaffolding role proposed for this kinase.

T rehalose is a non-reducing disaccharide found in bacteria, archaea, fungi, plants and some invertebrates ${ }^{1,2}$. Surprisingly, mammalian cellular systems show a complete lack of trehalose ${ }^{3}$. In mycobacteria, trehalose serves as a component of cell wall glycolipids, and plays an important role in cellular response to stress ${ }^{3}$. Notably, trehalose is constitutively present in the cytoplasm of mycobacteria and is constantly subject to turnover; exemplifying the importance of trehalose in mycobacterial metabolism ${ }^{4}$. A key conversion of trehalose to $\alpha$ glucans for the synthesis of the outer capsular layer and possibly methylglucose lipopolysaccharides contributes to the pathogenecity of Mycobacterium tuberculosis $(M t b)^{5,6}$. At least three different pathways are known to exist for the synthesis of $\alpha$-glucans. These include the GlgC-GlgA pathway ${ }^{6}$, the $\alpha(1->4)$ glucosyltransferase (Rv3032) pathway $^{6}$ and the TreS-MaK-GlgE-GlgB pathway ${ }^{7}$. These pathways have been shown to compensate each other for malfunctions in the pathways that stall $\alpha$-glucan synthesis. For example, $\Delta \mathrm{GlgE-} \Delta$ TreS mutants of mycobacterium with a deficient TreS-MaK-GlgE-GlgB pathway are viable ${ }^{8}$. When an additional loss-of-function mutation of Rv3032 was introduced in these mutants, they lost viability, highlighting the redundancy in pathways for synthesis of $\alpha$-glucans ${ }^{8}$.

Maltose kinase functions in the TreS-MaK-GlgE-GlgB pathway that has been shown to synthesize $\alpha$-glucans ${ }^{8}$. In this pathway, trehalose is first isomerized to maltose by trehalose synthase (TreS). This conversion is reversible $^{9,10}$. Next, maltose is phosphorylated to maltose-1-phosphate by maltose kinase (MaK) by expending a molecule of ATP. The phospho-activated disaccharide is a substrate for glycosyltransferase E (GlgE). GlgE uses maltose-1-phosphate to elongate $\alpha(1->4)$ linked glucan chains. The last enzyme of this pathway, glycosyltransferase $\mathrm{B}(\mathrm{G} \operatorname{lgB})$, mediates $\alpha(1->6)$-branching of the glucan chain ${ }^{11}$ (Supplementary Fig. S1). Interestingly, inactivation of GlgE leads to accumulation of maltose-1-phosphate, the product of MaK, during growth in presence of trehalose ${ }^{8}$. The accumulation of maltose-1-phosphate has been shown to be the cause of rapid death of $M t b$ in vitro and in mice ${ }^{8}$.

In vitro viability studies on $M t b$ subjected to transposon mutagenesis have helped identify mak as an essential gene for the growth of $M t b^{12,13}$. The product of MaK-mediated catalysis, maltose-1-phosphate, is used for synthesis of $\alpha$-glucans that make up almost $80 \%$ of the components of the outer capsule of $M t b^{14}$. However, unlike $M t b$, micro-organisms like Actinoplanes missouriensis and Streptomyces coelicolor that express MaK homologues do not produce outer capsular layer. In addition, MaK is constitutively expressed in Actinoplanes missouriensis ${ }^{15}$. These observations suggest the possibility of additional roles for maltose-1-phosphate other than synthesis of capsular $\alpha$-glucans. Biophysical characterization of $M t b \mathrm{MaK}$ using size exclusion chromatography and sedimentation velocity experiments suggests that $M t b \mathrm{MaK}$ exists as a mixture of monomers, trimers and tetramers in solution ${ }^{7}$. Notably, MaK forms a complex with TreS in vitro and in vivo ${ }^{7}$. This is not surprising partly 
due to the fact that the mak gene is usually linked with the treS gene and in some micro-organisms like Psuedomonas entamophila and Rubrobacter xylanophilus the two genes are fused into a single gene $\mathrm{e}^{16}$. Four units of $M t b \mathrm{MaK}$ associate with a tetramer of TreS to form a hetero-octameric complex ${ }^{7}$. More importantly, the formation of the complex enhances the activity of $M t b \mathrm{MaK}$ by 3 -folds ${ }^{7}$. Although the crystal structure of the tetramer of TreS is known ${ }^{7,17,18}$, the molecular basis for the enhancement of activity of MaK by TreS is currently not understood. In addition, the structural basis for conversion of maltose to maltose-1-phosphate is not known.

Here, we describe the crystal structures of the apo- and maltosebound $M t b \mathrm{MaK}$. Unexpectedly, the structures reveal a unique $\mathrm{N}$ lobe that has not been observed before in structures of other kinases. MaK uses homotypic dimerization to assemble the active sites for catalysis. Maltose binds in a spherical cavity of the C-lobe with the $\mathrm{O} 1^{\prime}$ oxygen atom oriented towards the catalytic D322. Instead of a glycine-rich loop, MaK uses a ${ }^{142} \mathrm{EQSNXS}^{147}$ motif conserved across MaK homologues to catalyse the phosphorylation of maltose. These structural variations and a putative role for the signal independent homotypic dimerization of $\mathrm{MaK}$ in scaffolding are discussed.

\section{Results}

Structure of MtbMaK reveals a unique N-lobe. Structure of the apoenzyme was solved by single-wavelength anomalous diffraction (SAD) method using crystals derivatized with $\mathrm{HgCl}_{2}$ (Table 1). The final model consists of amino acids 5-455, 154 water molecules, $1 \mathrm{SO}_{4}{ }^{2-}$ ion and 3 mercury atoms refined to $2.4 \AA$ resolution with an $\mathrm{R}$ factor of 0.207 and a free $\mathrm{R}$ value of 0.244 . The structure of maltose bound $M t b \mathrm{MaK}$ was solved by molecular replacement using the structure of the unliganded enzyme as a search template and consists of 450 amino acids, 1 molecule of maltose, 2 molecules of Bis-Tris, and $1 \mathrm{SO}_{4}{ }^{2-}$ ion refined to $2.9 \AA$ resolution with an $\mathrm{R}$ factor of 0.239 and a free $\mathrm{R}$ value of 0.281 .

The overall structure of $M t b \mathrm{MaK}$ can be divided into an N-lobe primarily composed of $\beta$ strands and a C-lobe made up of mostly $\alpha$ helices (Fig. 1a). Unlike typical kinases, the N-lobe of MaK contains not one, but two $\beta$-sheets. Each sheet encloses an $\alpha$ helix and a short $3_{10}$ helix. The sheet proximal to the $\mathrm{N}$-terminus of $M t b \mathrm{MaK}$ is highly twisted and is formed by three long $(\beta 1-\beta 3)$ and two short ( $\beta 4$ and $\beta 5)$ strands running anti-parallel to each other. In contrast, the second sheet is made of seven anti-parallel strands $(\beta 6-\beta 7, \beta 9-\beta 11$, $\left.\beta 8^{\prime}-\beta 9^{\prime}\right)$, that includes two strands $\left(\beta 8^{\prime}-\beta 9^{\prime}\right)$ contributed by an adjacent monomer. In exchange, strands $\beta 8$ and $\beta 9$ of this sheet protrude out of the protein, insert themselves into the adjacent monomer and make up two strands of the corresponding sheet in the adjacent monomer. This results in the formation of an unusual homotypic dimer of $M t b \mathrm{MaK}$ (Fig. 1b). The unique arrangement of the structural elements of the $\mathrm{N}$-lobe of $M t b \mathrm{MaK}$ constitutes a new fold. In addition, the signal independent homotypic dimerization of $M t b \mathrm{MaK}$ involving mutual exchange of structural elements from the $\mathrm{N}$-lobe, has not been observed before for any kinases. In contrast to the $\mathrm{N}$-lobe that is very different from the $\mathrm{N}$-lobes of most kinases, the overall structure of the C-lobe of $M t b \mathrm{MaK}$ resembles the C-lobes found in most kinases ${ }^{19}$.

Comparison with homologous kinases. 3D structure alignmentbased similarity search using Dali $^{20}$ retrieved methylthioribose (MTR) kinase (PDB code 2PUN; 13\% sequence identity, r.m.s. deviation of $3.9 \AA$ for 249 overlapping $\mathrm{C}_{\alpha}$ atoms; Fig. 1c) ${ }^{21}$ and choline kinase (PDB code $1 \mathrm{NW} 1 ; 16 \%$ sequence identity, r.m.s. deviation of $3.6 \AA$ for 228 overlapping $\mathrm{C}_{\alpha}$ atoms ${ }^{22}$ as structural matches. Other significant hits included aminoglycoside phosphotransferases

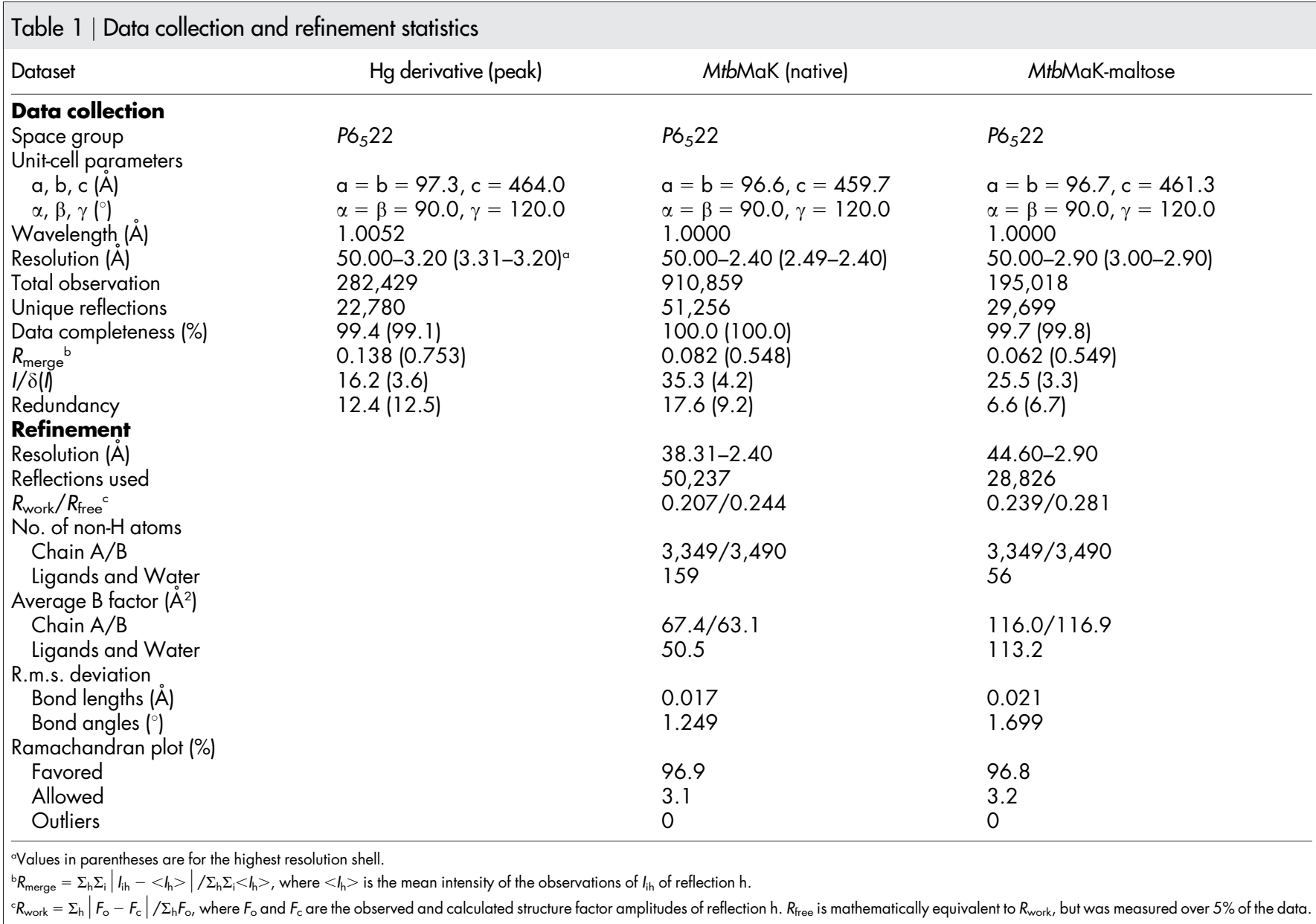



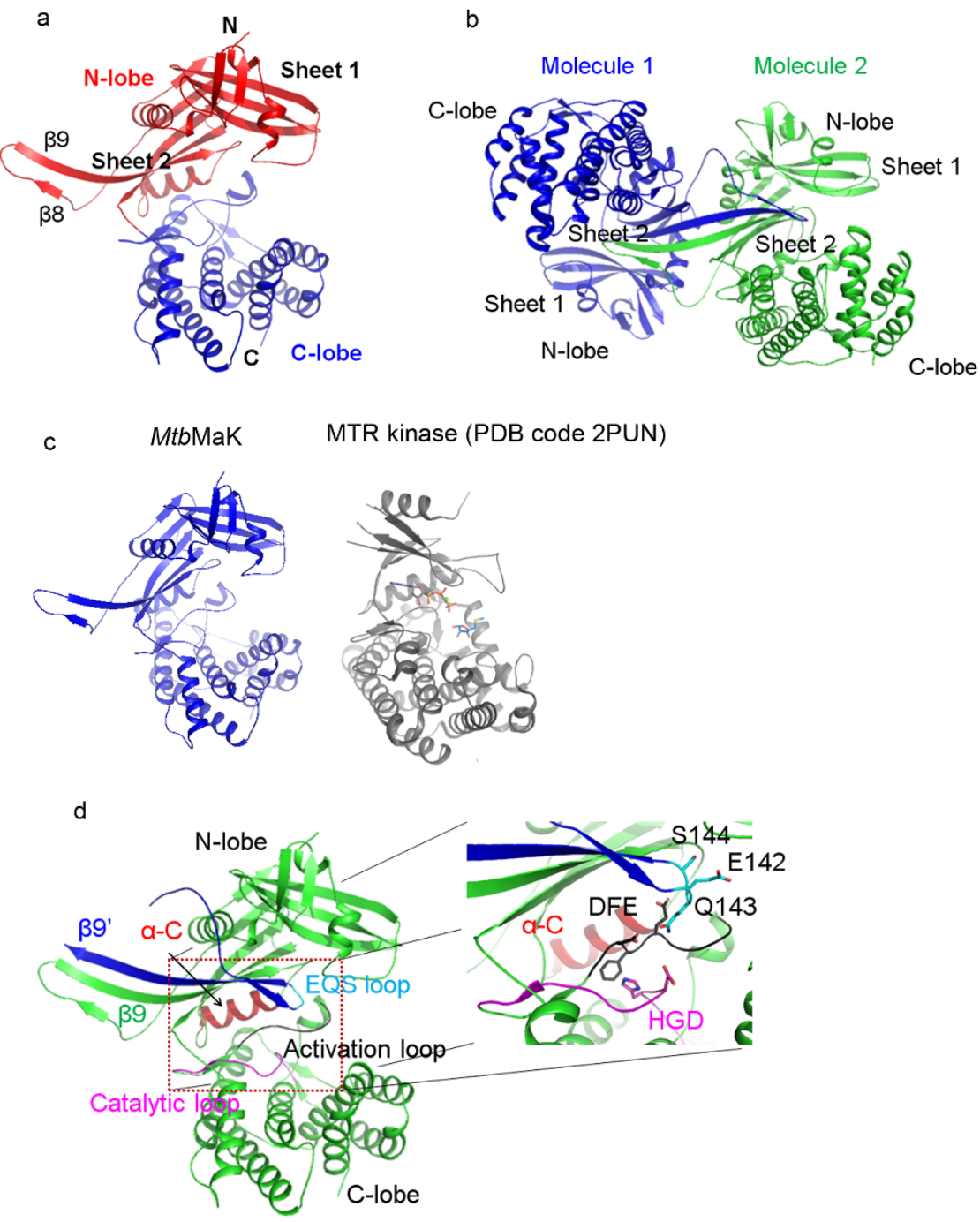

Figure $1 \mid$ Structure of MtbMaK. (a). Cartoon representation of a monomer of MtbMaK. The N-lobe contains two $\beta$-sheets with strands $\beta 8$ and $\beta 9$ protruding out of the protein. The $\mathrm{N}$ - and C-terminals of the protein are marked as $\mathrm{N}$ and $\mathrm{C}$, respectively. (b). Cartoon representation of a homotypic dimer of $M t b \mathrm{MaK}$. Strands $\beta 8$ and $\beta 9$ from each monomer within a dimer mutually insert into each other's N-lobe to assemble sheet 2. (c). Structural homologues of $M t b \mathrm{MaK}$. A Dali analysis of MtbMaK (left) retrieved low structural matches like methylthioribose (MTR) kinase from Bacillus subtilis (right; PDB code $2 \mathrm{PUN}$ ) that could not be superimposed over $M t b \mathrm{MaK}$. (d). Location of conserved motifs. The $\alpha$-C helix (shown in red), activation loop (black color) and catalytic loop (magenta color) of $M t b \mathrm{MaK}$ are located at the junction of $\mathrm{N}$ - and C-lobes. A ${ }^{142} \mathrm{EQS}^{144}$ loop (cyan color) connecting two strands of an adjacent monomer (blue color) contributes residues critical for catalysis. DFE and HGD motifs of $M t b M a K$ as well as residues of ${ }^{{ }^{142}} \mathrm{EQS}^{144 \text { ' }}$ loop are shown as sticks (inset).

and many eukaryotic protein kinases such as cell division protein kinase, casein kinase, proto-oncogene serine/threonine-protein kinase and cAMP-dependent protein kinase (PKA).

Since protein kinases have been characterized extensively and also because $M t b \mathrm{MaK}$ shares similarities with eukaryotic protein kinases, key features of $\mathrm{MtbMaK}$ are discussed in context with terminologies used for typical protein kinases. Comparisons with prokaryotic structural homologues like MTR and choline kinases bring out remarkable differences; illustrating $M t b \mathrm{MaK}$ has evolved specific features for its function. A common conserved feature found in most

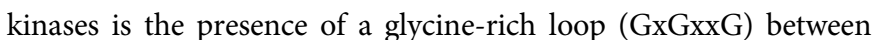
strands $\beta 1$ and $\beta 2$. Variations in composition of this loop that is involved in positioning of phosphate groups of ATP have been observed previously ${ }^{212}$. The structure of $M t b \mathrm{MaK}$ unveils a new variation, an ${ }^{142} \mathrm{EQS}^{144}$ sequence, at a structurally equivalent position (Fig. 1d). Interestingly, MTR kinase has a GxGNxN motif for binding $\mathrm{ATP}^{21}$ and choline kinase shows the presence of a di-glycine within the $\beta 1-\beta 2$ loop ${ }^{22}$. A lysine from $\beta 3$ strand of the $\mathrm{N}$-lobe usually makes contact with $\alpha$ and $\beta$ phosphates of ATP. K157 from strand $\beta 9$ of $M t b \mathrm{MaK}$ and K61 from strand $\beta 3$ of MTR kinase are located in equivalent positions. In choline kinase, R111 replaces the lysine at this position. Instead of a glutamate, D169 from the $\alpha \mathrm{C}$ helix of $M t b \mathrm{MaK}$ makes a salt bridge with $\mathrm{K} 157$, analogous to the salt bridge for the formation of an activated " $\alpha \mathrm{C}$-in" conformation of protein kinases. In case of MTR kinase, E84 located on $\alpha \mathrm{C}$ helix makes a typical salt bridge with K61. The corresponding glutamate E125 is dis-ordered in the structure of choline kinase. Absence of salt bridge signifies an inactive conformation. This is in agreement with the fact that choline kinase was crystallized in presence of $\mathrm{Ca}^{2+}$ ions that are known to inactivate the kinase ${ }^{12}$. The active site of kinases usually contain two aspartate residues; one each located on the catalytic and activation loops. D322 from the ${ }^{320} \mathrm{HGD}^{322}$ motif of $M t b \mathrm{MaK}$ is within hydrogen bonding distance of $\mathrm{O}^{\prime}$ ' hydroxyl oxygen of maltose and can function as the key catalytic residue. D233 of MTR kinase located within a similar HGD motif makes contact with $\mathrm{O}^{\prime}$ hydroxyl oxygen of the substrate. Intriguingly, choline kinase has a 


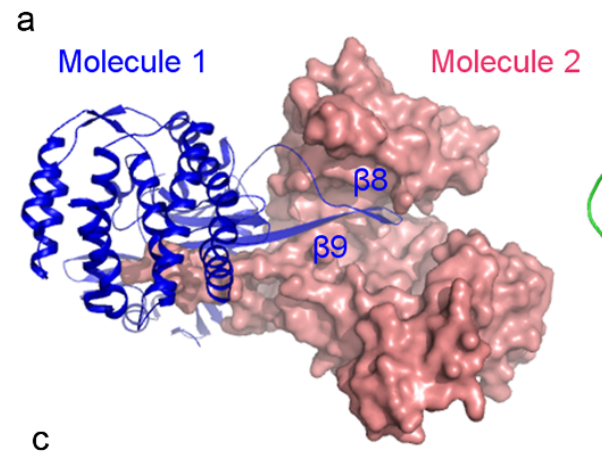

b

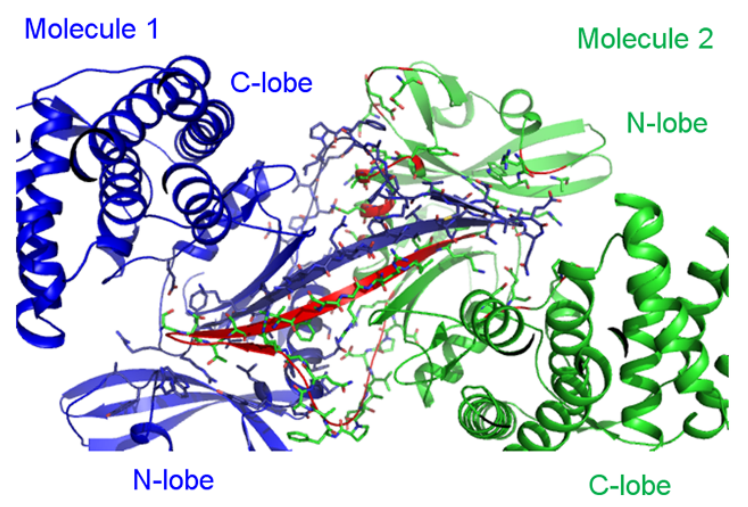

Figure $2 \mid$ Homotypic dimerization of $\mathbf{M t b M a K}$. (a). A dimer of $M t b \mathrm{MaK}$ where one molecule is depicted as cartoon (blue color), while the second molecule is shown in a surface representation (salmon color). Mutual insertion of strands $\beta 8$ and $\beta 9$ results in the formation of a tight dimer. (b). Strand $\beta 9$ of each monomer (green and blue) run anti-parallel with the backbone atoms interacting along the entire length of the strand. (c). Dimer interface of $\mathrm{MtbMaK}$. Interfacing residues are shown in sticks. Region of molecule 2 participating in dimerization is marked with red color on the cartoon. Side chains as well as backbone atoms participate in dimerization.

HND motif with D255 occupying a catalytically equivalent position. The second aspartate of the active site is part of the activation loop that positions the $\mathrm{Mg}^{2+}$ ions for catalysis. D339 of $M t b \mathrm{MaK}$ from the ${ }^{339} \mathrm{DFE}^{341}$ motif, D250 of MTR kinase and D301 of choline kinase located within a DXE motif are well positioned to perform this role. Thus, although $M t b \mathrm{MaK}$ shares many catalytic features with other kinases, key differences like presence of the 'EQS' loop, which is surprisingly widely distributed in MaK homologues, suggests function-specific alterations of the kinase.

$M t b M a K$ exhibits a unique mode of dimerization. Analysis of the asymmetric unit of the crystallized $M t b \mathrm{MaK}$ revealed the presence of two molecules of the protein. These two molecules of $M t b \mathrm{MaK}$ form a dimer via homotypic interactions that involve backbone atoms as well as side chains. Strands $\beta 8$ and $\beta 9$ from one monomer protrude out and insert into the active site of another monomer (Fig. 2a). Amino acids N145-F159 making up strand $\beta 9$ of the monomers within a dimer run anti-parallel with the backbone atoms from both the strands interacting along the entire length of the strands (Fig. 2b). Additional intermolecular interactions are observed between side chains of residues from the ${ }^{142} \mathrm{EQS}^{144}$ loop and the loop connecting helix $\alpha 1$ with strand $\beta 1$. Interestingly, intermolecular interactions between the ${ }^{142} \mathrm{EQS}^{144}$ loop and the "activation loop" are also observed. Further, the loop connecting strand $\beta 7$ with $\beta 8$ interacts with residues connecting strands $\beta 3$ with $\beta 4$ and helix $\alpha 2$ (Fig. 2c). Details of amino acids involved in inter-molecular interactions are listed in Table S1. PISA ${ }^{23}$ analysis revealed that dimerization of $M t b \mathrm{MaK}$ buries 2,575 $\AA^{2}$ of solvent accessible surface area. Intriguingly, structural matches like MTR kinase, choline kinase and aminoglycoside phosphotransferase type IIIa (APH IIIa) retrieved by Dali analysis for $M t b \mathrm{MaK}$, are also known to form dimers ${ }^{21,22,24}$. However, dimerization of these kinases involves participation of C-lobe of one of the monomers. On the other hand, crystal structure of the kinase domain of a transmembrane Thr/Ser kinase, PknB (PDB code $1 \mathrm{MRU})^{25}$, does show the Nlobe forming intermolecular interactions. But this mode of dimerization mediated by the N-lobe is very different from that of MaK. While dimerization involves surface residues in $\mathrm{PknB}$, structural elements are mutually exchanged between the N-lobes of two monomers of MaK. As a result, dimerization of $M t b \mathrm{MaK}$ buries a much larger solvent accessible surface area $\left(2,575 \AA^{2}\right)$ when compared to that buried during dimerization of $\operatorname{PknB}\left(1,045 \AA^{2}\right)$. Dimerization involving mutual exchange of structural elements has been seen previously in human ChPK2 (Supplementary Fig. S2) ${ }^{26}$. However, ChPK2 dimerization involves the transactivation loop (Tloop) of the C-lobe and not the N-lobe. Thus, the mode of dimerization of $M t b \mathrm{MaK}$ is unique. Extensive inter-subunit interactions result in the formation of a tight dimer of $M t b \mathrm{MaK}$. This dimerization of $M t b \mathrm{MaK}$ is signal independent and involves homotypic interactions.

Maltose-binding site of $M t b M a K$. To gain insights into the nature of maltose binding site, we solved the structure of $M t b \mathrm{MaK}$ in complex with maltose. As observed for the unliganded enzyme, MtbMaK bound with maltose crystallized as a dimer. Electron density for maltose in Chain B was unambiguous (Fig. 3a). Maltose binding induces conformational changes around the substrate binding site. However, no movement of the lobes is observed. Side chains of amino acids like Y370, Y429, E430, Y433 and R438 move inwards to interact with maltose (Fig. 3b). Detailed analysis of the substratebinding pocket revealed that 11 residues, W222, D322, H324, P344, S367, Y370, K426, Y429, E430, Y433 and R438, are interacting with maltose (Fig. 3c-e and Supplementary Table S2). These interactions fix the orientation of maltose for catalysis. Notably, the OD2 carboxyl oxygen of the catalytic D322 is observed forming a hydrogen bond with the $\mathrm{O}^{\prime}$ atom of maltose. $\mathrm{MtbMaK}$ exhibited a $\mathrm{Km}$ value of 
a

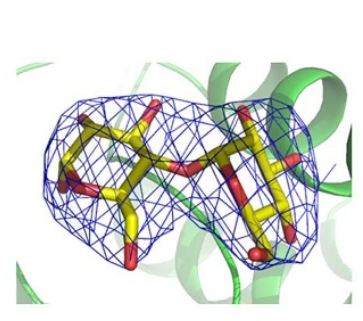

d

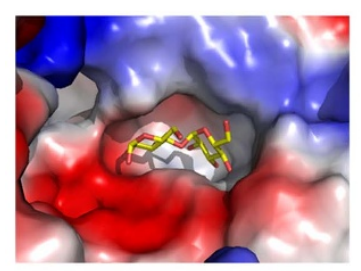

b
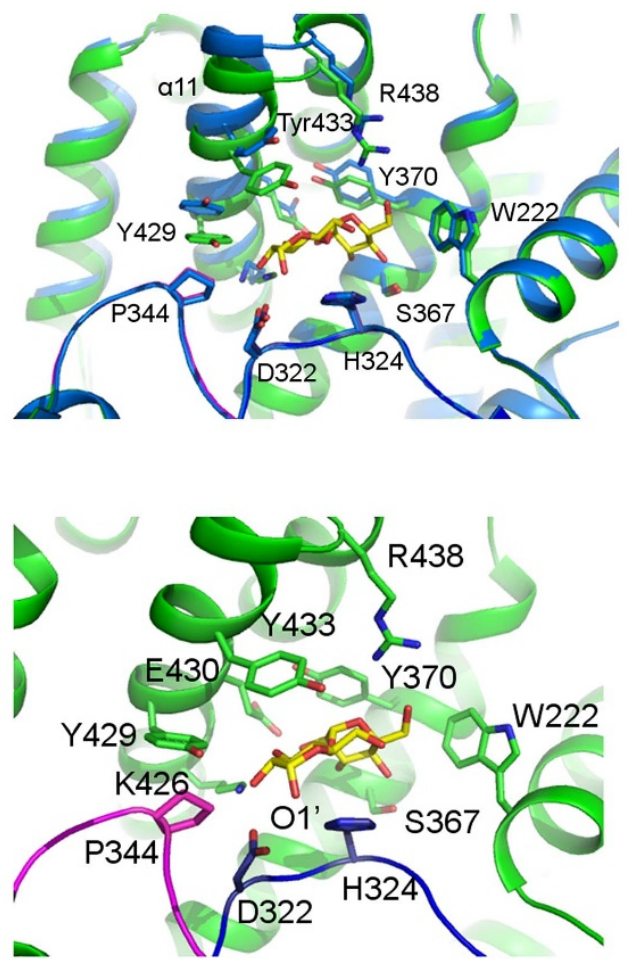

C

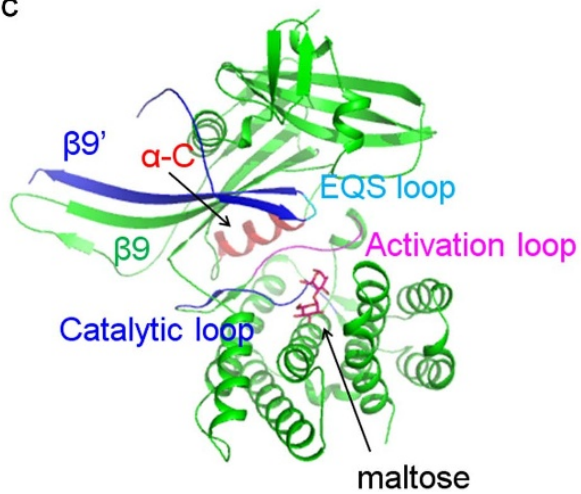

f

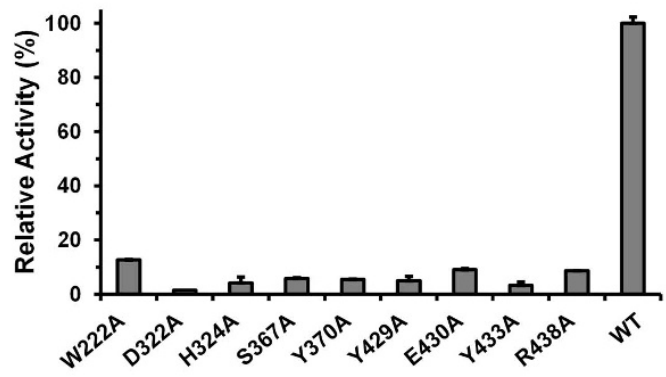

Figure $3 \mid$ Maltose binding site of MtbMaK. (a). 2Fo-Fc electron density for maltose contoured at $1.5 \sigma$ is shown. (b). Conformational changes upon binding of maltose. The structure of maltose bound $M t b \mathrm{MaK}$ (green color) was superimposed over the unliganded structure (blue color). Main and side chains of several residues move as a result of substrate binding. (c). Maltose binds in the C-lobe and is in proximity to the conserved motifs essential for catalysis. (d). A surface electrostatic potential representation of the region around the maltose binding site. Blue represents positive potential; red, negative potential. (e). Residues interacting with maltose. Residues from the catalytic loop interacting with maltose are shown as blue sticks, while P344 from the activation loop is shown as magenta sticks. Maltose is shown as sticks in panels A-E. (f). Alanine scanning mutagenesis of amino acids interacting with maltose. Relative activity of mutants (\%) with respect to the wild type is plotted as a bar graph. Error bars represent s.d. $(\mathrm{n}=3$ ).

$2.49 \mathrm{mM}$ for maltose (Fig. S7). This value is similar to the previously reported value for $M$. bovis $\mathrm{BCG} \mathrm{MaK}(2.52 \mathrm{mM})^{16}$. To verify the role of the amino acids interacting with maltose in catalysis, we performed alanine-scanning mutagenesis and tested the ability of the mutants to phosphorylate maltose. Except for the P344A and K426A mutants, all other mutants could be expressed as soluble protein in E. coli. All nine mutants exhibited a dramatic loss in activity (activity less than 13\% of the wild type) (Fig. $3 \mathrm{f}$ and Supplementary Table S4). Taken together, the structural observations coupled with results of mutagenesis studies suggest that Y222, D322, H324, S367, Y370, Y429, E430, Y433 and R438 are crucial for phosphorylation of maltose. These amino acids are conserved across MaK homologues from different sources (Supplementary Fig. S3).

The structure of the binary complex of $M t b \mathrm{MaK}$ with maltose helps explain the structural basis for $\alpha$ maltose-specific (Supplementary Fig. S4) phosphorylation by MaK. The aromatic ring of Y433 stacks above the glycosyl moiety with the reducing end (Fig. 3e). Specifically, the $\mathrm{C} 1$ atom of the sugar is $3.5 \AA$ away from the aromatic ring. In its $\beta$ form, the $\mathrm{O} 1$ atom of maltose would either sterically clash with the aromatic ring of Y433 or result in non-productive binding. This is because stacking of Y433 with the glycosyl moiety brings catalytically critical residues in proximity of maltose (Fig. 3b). A $\beta$ form of the maltose would not permit this stacking. In addition, the catalytic machinery is positioned for phosphorylation of the $\mathrm{O}^{\prime}$ atom of the $\alpha$ isomer. The $\mathrm{O}^{\prime}$ atom of maltose's $\beta$ form would be positioned far away from D322 to permit phosphorylation at this position.
Nucleotide-binding site of $\mathbf{M t b M a K}$. Although there is no significant sequence conservation between $M t b \mathrm{MaK}$ and its homologues over the entire length of the protein, some conservation around the ATP-binding site is observed (Fig. 4a). To gain insights into the ATP-binding site of $M t b \mathrm{MaK}$, the nucleotide-binding site of aminoglycoside phosphotransferase (APH; PDB code 1J7U) was superimposed over the structure of $M t b \mathrm{MaK}$. This region of $\mathrm{APH}$ superimposed over an equivalent region of $M t b \mathrm{MaK}$ with an r.m.s.d. of $2.6 \AA$ between the $\mathrm{C}_{\alpha}$ atoms of 90 matching residues. Using such an approach, AMPPNP-Mg of APH could be docked into the nucleotide-binding site of $M t b \mathrm{MaK}$ (Fig. 4b) and a putative mode of ATP binding by $M t b \mathrm{MaK}$ could be inferred. The mode of ATP binding was verified by performing alanine-scanning mutagenesis of residues of $M t b \mathrm{MaK}$ interacting with the docked AMPPNP-Mg moiety (Supplemental text; Fig. 4c and Supplementary Table S4). The analysis revealed that amino acids like E142, Q143 and S144 from the "EQS" loop that is conserved across all MaK homologues (Supplementary Fig. S3) are important for binding ATP.

\section{Discussion}

Our structural studies on $M t b \mathrm{MaK}$ unveil several new, previously unknown features of maltose kinases. Many kinases dimerize upon receiving a signal and activate cellular signaling pathways ${ }^{27}$. Such dimerization usually involves the C-lobe ${ }^{28}$. In contrast, $M t b \mathrm{MaK}$ undergoes signal-independent homotypic dimerization mediated by the N-lobe. Dimerization probably offers a means for $M t b \mathrm{MaK}$ to form a scaffold that could readily hetero-oligomerize with the 
a

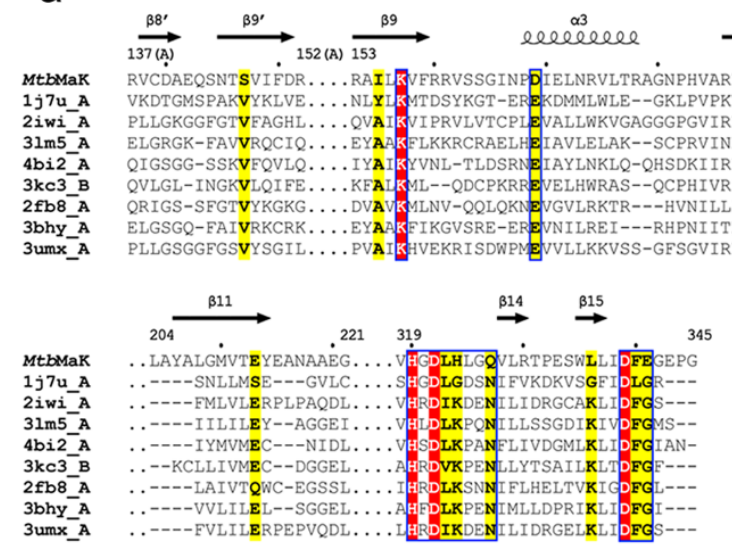

C

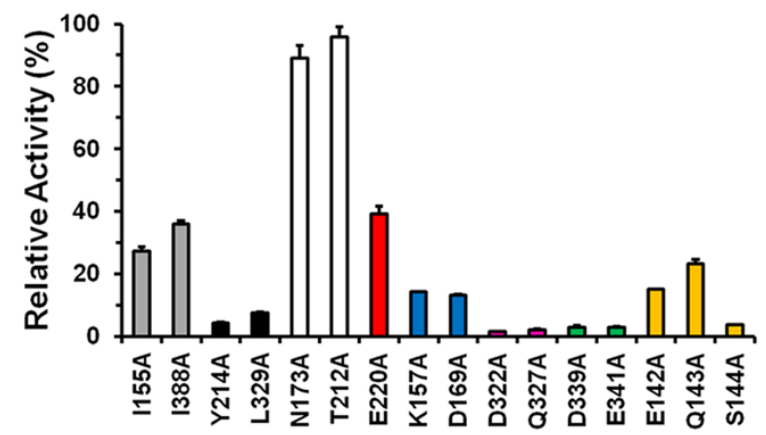

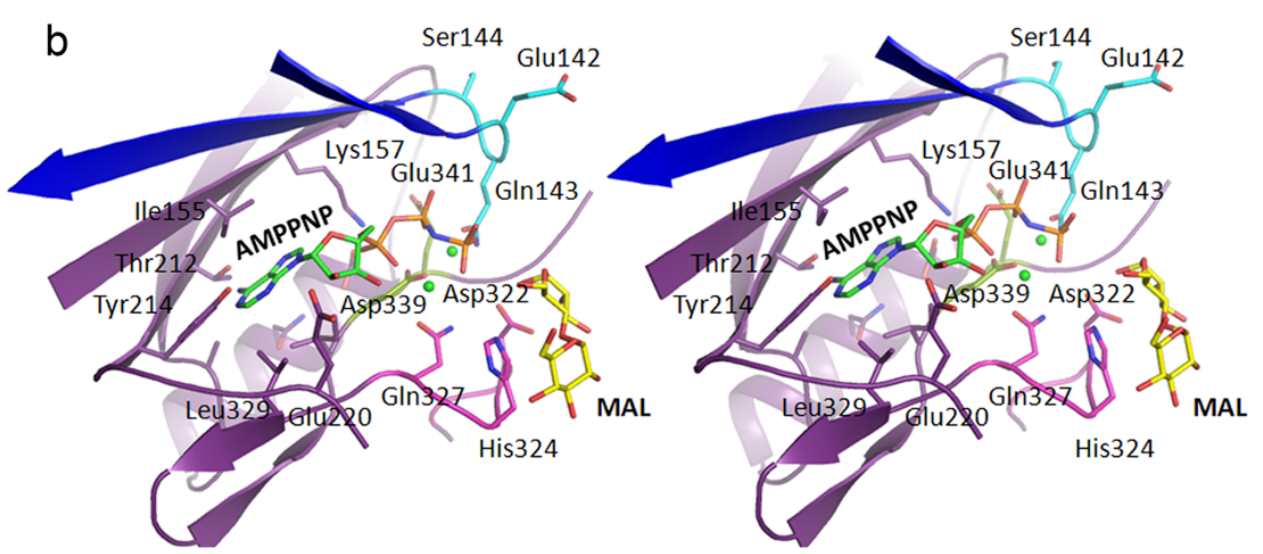

MtbTres tetramer

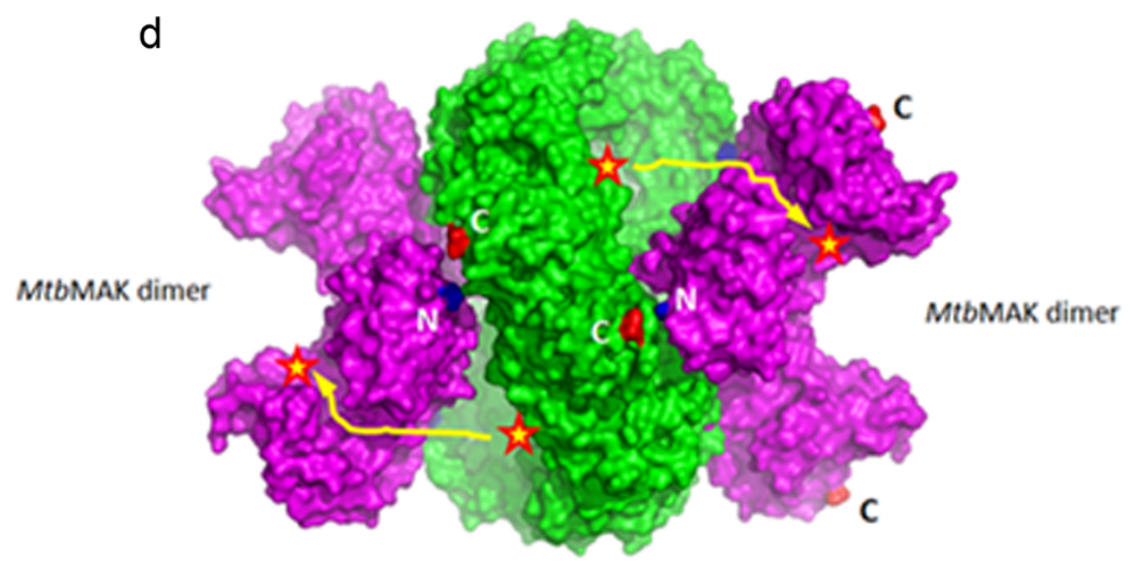

Figure $4 \mid$ Nucleotide binding site of $M t b M a K$. (a). Sequence alignment of nucleotide binding site (NBS) of MtbMaK and its homologues. SSE of $M t b \mathrm{MaK}$ are labeled on top of the aligned sequences. Identical residues are highlighted in red, and other conserved residues are highlighted in yellow. (b). Stereo view of AMPPNP-Mg (green sticks and spheres, PDB code $1 \mathrm{J7U}$ ) superimposed on the NBS of MtbMaK. The C atoms of ${ }^{(142} \mathrm{EQS}^{144}$ ' loop, HGD motif and DFE motif are colored in cyan, magenta and light green, respectively. Strands $\beta 8^{\prime}$ and $\beta 9^{\prime}$ from the other subunit of $M t b \mathrm{MaK}$ are colored in blue. The side chains of residues potentially interacting with AMPPNP-Mg are shown as sticks. The $\mathrm{O}$ and $\mathrm{N}$ atoms are colored in red and blue. (c). Mutagenesis of residues from the NBS. A bar graph of relative activity (\%) of mutants compared to the wild type enzyme is shown. Error bars represent s.d. $(\mathrm{n}=3)$. (d). Model of hetero-octameric complex of TreS with MaK. Potential interaction of a tetramer of TreS (green color, PDB code $4 \mathrm{LXF}$ ) with two dimers of $\mathrm{Mtb \textrm {MaK }}$ (purple color) is shown. Active sites are marked with a red star; putative path of product marked in yellow.

upstream enzyme, TreS, of the TreS-MaK-GlgE-GlgB pathway (Supplementary Fig. S1). The premises for such a scaffolding role for $M t b \mathrm{MaK}$ can be found in the hetero-octameric complex of TreS-MaK reported previously ${ }^{7}$. Further support for the scaffolding role of $\mathrm{MaK}$ is provided by the fact that the mak gene is usually located immediately downstream of the tres gene and in some micro-organisms the two genes are fused into a single gene ${ }^{16}$. Using shape complementarity, a model for interaction of MaK with TreS could be built with the $\mathrm{N}$-terminus of MaK placed in proximity of the C-terminus of TreS, depicting the closely linked nature and order of the genes. In this model, a dimer of MaK fits on each face of a tetramer of TreS (PDB code 4LXF; Fig. 4d). Such an arrangement of 
TreS-MaK subunits aligns the active sites of the two proteins in a manner that facilitates the transfer of the product of TreS, maltose, into the active site of MaK for phosphorylation (Fig. 4d).

Scaffolding roles for kinases have been demonstrated previously. For example, the integrin-linked kinase (ILK) functions exclusively as a scaffolding kinase. ILK binds $\mathrm{CH} 2$ domain of $\alpha$-parvin and integrin tails during recruitment of focal adhesion proteins ${ }^{29}$. However, ILK cannot catalyze phospho-transfers due to a defunct active site. In contrast, $M t b \mathrm{MaK}$ is active and readily phosphorylates $\alpha$-maltose. Since maltose-1-phosphate is toxic to mycobacterium, it needs to be converted into other metabolites quickly. This could be accomplished by assembling a super complex of TreS-MaK-GlgE as proposed previously ${ }^{7}$. Using shape complementarity a dimer of GlgE could be possibly fit on the un-occupied face of the MaK dimer (Fig. 4d) giving rise to a super complex in the order GlgE dimer MaK dimer - Tres tetramer - MaK dimer - GlgE dimer. Thus, MaK kinase could possibly serve as a scaffold for the assembly of a super complex. However, biochemical evidence for a TreS-MaK-GlgE complex has not come forth as yet. Results of our preliminary studies on ability of $M t b \mathrm{MaK}$ to form a complex with $M t b$ GlgE using GSTpulldown assays and in vitro binding studies of tag-less protein using Biacore suggests that unliganded recombinant proteins do not form a complex. Perhaps, additional factors like ligands or TreS are required for the formation of the complex.

The dimer of $M t b \mathrm{MaK}$ observed in the crystal structure probably represents only one of the conformations assumed by the protein. $M t b \mathrm{MaK}$ eluted in two peaks during size exclusion chromatography (SEC) runs. Subsequent HPLC analysis revealed a similar pattern (Supplementary Fig. S5A). The retention time of the larger peak suggested presence of a dimer of $\mathrm{MaK}$, while the shorter peak consisted of monomers of MaK. Protein from both the peaks was active when tested for kinase activity (Supplementary Fig. S5B). Analysis of symmetry mates revealed that $M t b M a K$ could possibly form a tetramer (Supplementary Fig. S6). PISA analysis suggested a Complexation Significance Score (CSS) of 0.769 for the tetrameric assembly, indicating the possibility of $M t b \mathrm{MaK}$ assuming a tetrameric biological assembly. These results are consistent with previously reported ability of $M t b \mathrm{MaK}$ to exist in different oligomeric states in solution ${ }^{7}$.

The structures of $M t b \mathrm{MaK}$ reported here could be used as guides to develop inhibitors that could potentially aid treatment of TB. Analogues of $\alpha$-maltose and maltose-1-phosphate could serve as scaffolds that could be re-designed to increase potency and other drug-like properties. Further, the use of MaK inhibitors could be extended to control other pathogens like Pseudomonas spp. that harbor the conserved mak gene. In this context, lack of mak gene in mammalian systems could prove to be beneficial for achieving greater specificity.

\section{Methods}

Molecular cloning. The gene encoding the full length $M t b \mathrm{MaK}$ (Rv0127) was amplified by polymerase chain reaction (PCR) from Mycobacterium tuberculosis H37Rv genomic DNA. The product was sub-cloned into expression vector pGEX-6p1 (GE Lifesciences) after digestion with $\mathrm{BamHI}$ and $\mathrm{XhoI}(\mathrm{TaKaRa})$. Site-directed mutagenesis was performed by PCR using native $M t b \mathrm{MaK}$ expression plasmid as a template. All mutants were constructed as per manufacturer's instructions. Clones were verified by sequencing the entire gene. Recombinant plasmid containing either native mak gene or the mutants was then transformed into E. coli BL21 (DE3) for protein expression. Primers used in molecular cloning are listed in Supplementary Table S3.

Protein expression and purification. Cells containing recombinant plasmid were grown in Luria Bertani medium at $37^{\circ} \mathrm{C}$ until $\mathrm{OD}_{600 \mathrm{~nm}}$ reached 0.8 . After cooling the culture, GST-tagged $M t b \mathrm{MaK}$ protein expression was induced at $16^{\circ} \mathrm{C}$ by addition of $0.5 \mathrm{mM}$ Isopropyl $\beta$-D-thiogalactoside (IPTG). After $20 \mathrm{~h}$ of induction, cells were harvested by centrifugation and re-suspended in $1 \times \mathrm{PBS}, \mathrm{pH} 7.4 . \mathrm{MtbMaK}$ was recovered by first lysing the cells using sonication and clarifying the lysate by centrifugation at $30,000 \mathrm{~g}$ for $30 \mathrm{~min}$ to remove cell debris. The supernatant containing soluble recombinant protein was subjected to GST-affinity chromatography. Glutathione Sepharose $4 \mathrm{~B}$ beads mixed with the supernatant were washed with buffer to remove non-specifically bound proteins. To remove the GST tag, PreScission protease (GE Lifesciences) was added and the mixture incubated overnight at $4^{\circ} \mathrm{C}$. Tagless $M t b \mathrm{MaK}$ protein was eluted from the column by gravity, concentrated and injected on a Superdex 200 10/300 GL gel filtration column equilibrated with a buffer containing $20 \mathrm{mM}$ Tris- $\mathrm{HCl}, \mathrm{pH} 8.0$, and $150 \mathrm{mM} \mathrm{NaCl}$. Fractions containing the dimer were pooled and concentrated to $10 \mathrm{mg} / \mathrm{mL}$ for crystallization. Mutant proteins were expressed and purified in a similar way.

Crystallization and structure determination. Crystallization screening experiments were first carried out at $16^{\circ} \mathrm{C}$ by vapor diffusion method in 96-well plates using a Mosquito liquid handling system (TTP Labtech). Each crystallization drop contained $200 \mathrm{~nL}$ of $M t b \mathrm{MaK}$ protein $(10 \mathrm{mg} / \mathrm{mL})$ mixed with $200 \mathrm{~nL}$ of reservoir solution. Commercially available sparse matrix screens were used for screening crystallization conditions. Crystals grew in a solution containing $1 \mathrm{M}\left(\mathrm{NH}_{4}\right)_{2} \mathrm{SO}_{4}, 0.1 \mathrm{M}$ Bis-Tris, $\mathrm{pH} 5.5$, and $1 \%(\mathrm{w} / \mathrm{v})$ PEG3350. Crystals were optimized by varying the concentration of $\left(\mathrm{NH}_{4}\right)_{2} \mathrm{SO}_{4}$ and $\mathrm{pH}$. Optimized crystals were soaked in the reservoir solution supplemented with $20 \%$ (v/v) glycerol for $10 \mathrm{~s}$ and flash frozen in liquid nitrogen. Xray diffraction data were collected at $100 \mathrm{~K}$ on beamline BL17U at SSRF (Shanghai, China) and processed using HKL2000 (HKL Research, Inc.) ${ }^{30}$. Mercury derivatives were prepared by soaking the native crystals in the reservoir solution supplemented with $1 \mathrm{mM} \mathrm{HgCl}_{2}$ for $2 \mathrm{~h}$ prior to cryoprotection. Anomalous peak data were collected on beamline BL41XU at Spring-8 (Hyogo, Japan) and processed to $3.2 \AA$ resolution. The structure of $M t b \mathrm{MaK}$ was determined by the single-wavelength anomalous diffraction (SAD) method, using the peak data. All the 3 potential mercury atoms covalently linked to three cysteine residues were located using SHELXD $^{31}$, and the initial phases were calculated using PHENIX ${ }^{32}$. The structural model was initially built according to the electron density map and then refined using the native data collected to $2.4 \AA$ resolution. Manual adjustments to the model were made using COOT $^{33}$ and refined using PHENIX. The MtbMaK-maltose complex was prepared by soaking $M t b \mathrm{MaK}$ crystals in the reservoir solution supplemented with $10 \mathrm{mM}$ maltose (Sigma-Aldrich) for $1 \mathrm{~h}$. The diffraction data for the complex were collected on beamline BL5A at KEK (Tsukuba, Japan) and processed to $2.9 \AA$ resolution. The $M t b \mathrm{MaK}$-maltose complex structure was solved by the molecular replacement (MR) method with Phaser program ${ }^{34}$ in $\mathrm{CCP} 4^{35}$, using the native structure as a search template. The complex structure was refined using the native structure as a reference model in order to restrain the dihedral angles. The final structural models of native $M t b \mathrm{MaK}$ and $M t b \mathrm{MaK}$-maltose complex have $R_{\text {work }} / R_{\text {free }}$ values of $0.207 / 0.244$ and $0.239 / 0.281$, respectively. Data collection and refinement statistics are listed in Table 1. Structural figures were prepared using PyMol (DeLano Scientific).

Enzyme activity assay. Kinase activity was estimated by a linked spectrophotometric assay that uses pyruvate kinase and L-lactate dehydrogenase as auxiliary enzymes to monitor the conversion of ATP to ADP with an observable decrease in the absorption of $\mathrm{NADH}$ at $340 \mathrm{~nm}^{36} .100 \mu \mathrm{L}$ of the reaction mixture contained $2 \mu \mathrm{g} \mathrm{MtbMaK}$, $10 \mathrm{mM} \mathrm{MgCl}_{2}, 1.5 \mathrm{mM}$ ATP, and $5 \mathrm{mM}$ maltose suspended in a buffer made up of $20 \mathrm{mM}$ Tris- $\mathrm{HCl}, \mathrm{pH} 8.0,150 \mathrm{mM} \mathrm{NaCl}$. $3 \mathrm{U}$ of pyruvate kinase, $3 \mathrm{U}$ of L-lactate dehydrogenase, $0.3 \mathrm{mM}$ NADH and $2.5 \mathrm{mM}$ phosphoenolpyruvate (all from SigmaAldrich), were added and the mixture incubated at $37^{\circ} \mathrm{C}$ for $30 \mathrm{~min}$. The change in $\mathrm{A}_{340 \mathrm{~nm}}$ value was measured using a VarioshanFlash spectrophotometer (Thermo Scientific) over the course of the incubation period. The velocity of formation of the product ADP was calculated. The $K m$ value of $M t b \mathrm{MaK}$ for maltose was determined from the Lineweaver-Burk plot constructed by measuring enzyme activity using different concentrations of the substrate.

1. Iturriaga, G., Suarez, R. \& Nova-Franco, B. Trehalose metabolism: from osmoprotection to signaling. Int J Mol Sci 10, 3793-3810 (2009).

2. De Smet, K. A., Weston, A., Brown, I. N., Young, D. B. \& Robertson, B. D. Three pathways for trehalose biosynthesis in mycobacteria. Microbiology 146 (Pt1), 199-208 (2000).

3. Elbein, A. D., Pan, Y. T., Pastuszak, I. \& Carroll, D. New insights on trehalose: a multifunctional molecule. Glycobiology 13, 17R-27R (2003).

4. Winder, F. G., Tighe, J. J. \& Brennan, P. J. Turnover of acylglucose, acyltrehalose and free trehalose during growth of Mycobacterium smegmatis on glucose. J Gen Microbiol 73, 539-546 (1972).

5. Mendes, V., Maranha, A., Alarico, S. \& Empadinhas, N. Biosynthesis of mycobacterial methylglucose lipopolysaccharides. Nat Prod Rep 29, 834-844 (2012).

6. Sambou, T. et al. Capsular glucan and intracellular glycogen of Mycobacterium tuberculosis: biosynthesis and impact on the persistence in mice. Mol Microbiol 70, 762-774 (2008).

7. Roy, R. et al. Synthesis of alpha-Glucan in Mycobacteria Involves a Heterooctameric Complex of Trehalose Synthase TreS and Maltokinase Pep2. ACS Chem Biol 8, 2245-2255 (2013).

8. Kalscheuer, R. et al. Self-poisoning of Mycobacterium tuberculosis by targeting GlgE in an alpha-glucan pathway. Nat Chem Biol 6, 376-384 (2010).

9. Pan, Y. T. et al. Trehalose synthase converts glycogen to trehalose. FEBS J 275, 3408-3420 (2008).

10. Elbein, A. D., Pastuszak, I., Tackett, A. J., Wilson, T. \& Pan, Y. T. Last step in the conversion of trehalose to glycogen: a mycobacterial enzyme that transfers 
maltose from maltose 1-phosphate to glycogen. J Biol Chem 285, 9803-9812 (2010).

11. Pal, K. et al. Crystal structure of full-length Mycobacterium tuberculosis H37Rv glycogen branching enzyme: insights of N-terminal beta-sandwich in substrate specificity and enzymatic activity. J Biol Chem 285, 20897-20903 (2010).

12. Sassetti, C. M., Boyd, D. H. \& Rubin, E. J. Genes required for mycobacterial growth defined by high density mutagenesis. Mol Microbiol 48, 77-84 (2003).

13. Griffin, J. E. et al. High-resolution phenotypic profiling defines genes essential for mycobacterial growth and cholesterol catabolism. PLoS Pathog 7, e1002251 (2011).

14. Sani, M. et al. Direct visualization by cryo-EM of the mycobacterial capsular layer: a labile structure containing ESX-1-secreted proteins. PLoS Pathog 6, e1000794 (2010).

15. Niehues, B. et al. Isolation and characterization of maltokinase (ATP:maltose 1phosphotransferase) from Actinoplanes missouriensis. Arch Microbiol 180, 233-239 (2003).

16. Mendes, V., Maranha, A., Lamosa, P., da Costa, M. S. \& Empadinhas, N. Biochemical characterization of the maltokinase from Mycobacterium bovis BCG. BMC Biochem 11, 21 (2010).

17. Caner, S. et al. The structure of the Mycobacterium smegmatis trehalose synthase reveals an unusual active site configuration and acarbose-binding mode. Glycobiology 23, 1075-1083 (2013).

18. Ravaud, S. et al. Trehalulose synthase native and carbohydrate complexed structures provide insights into sucrose isomerization. J Biol Chem 282, 28126-28136 (2007)

19. Taylor, S. S. \& Kornev, A. P. Protein kinases: evolution of dynamic regulatory proteins. Trends Biochem Sci 36, 65-77 (2011).

20. Holm, L. \& Rosenstrom, P. Dali server: conservation mapping in 3D. Nucleic Acids Res 38, W545-549 (2010).

21. Ku, S. Y. et al. Structures of 5-methylthioribose kinase reveal substrate specificity and unusual mode of nucleotide binding. J Biol Chem 282, 22195-22206 (2007).

22. Peisach, D., Gee, P., Kent, C. \& Xu, Z. The crystal structure of choline kinase reveals a eukaryotic protein kinase fold. Structure 11, 703-713 (2003).

23. Krissinel, E. \& Henrick, K. Inference of macromolecular assemblies from crystalline state. J Mol Biol 372, 774-797 (2007).

24. Burk, D. L., Hon, W. C., Leung, A. K. \& Berghuis, A. M. Structural analyses of nucleotide binding to an aminoglycoside phosphotransferase. Biochemistry $\mathbf{4 0}$, 8756-8764 (2001).

25. Young, T. A., Delagoutte, B., Endrizzi, J. A., Falick, A. M. \& Alber, T. Structure of Mycobacterium tuberculosis PknB supports a universal activation mechanism for Ser/Thr protein kinases. Nat Struct Biol 10, 168-174 (2003).

26. Oliver, A. W. et al. Trans-activation of the DNA-damage signalling protein kinase Chk2 by T-loop exchange. EMBO J 25, 3179-3190 (2006).

27. Lassila, J. K., Zalatan, J. G. \& Herschlag, D. Biological phosphoryl-transfer reactions: understanding mechanism and catalysis. Annu Rev Biochem 80, 669-702 (2011).

28. Bartek, J. \& Lukas, J. DNA damage checkpoints: from initiation to recovery or adaptation. Curr Opin Cell Biol 19, 238-245 (2007).

29. Fukuda, K., Gupta, S., Chen, K., Wu, C. \& Qin, J. The pseudoactive site of ILK is essential for its binding to alpha-Parvin and localization to focal adhesions. Mol Cell 36, 819-830 (2009).
30. Otwinowski, Z. \& Minor, W. Processing of $\mathrm{x}$-ray diffraction data collected in oscillation mode. Methods Enzymol 276, 307-326 (1997).

31. Schneider, T. R. \& Sheldrick, G. M. Substructure solution with SHELXD. Acta Crystallogr D Biol Crystallogr 58, 1772-1779 (2002).

32. Echols, N. et al. Graphical tools for macromolecular crystallography in PHENIX. J Appl Crystallogr 45, 581-586 (2012).

33. Emsley, P. \& Cowtan, K. Coot: model-building tools for molecular graphics. Acta Crystallogr D Biol Crystallogr 60, 2126-2132 (2004).

34. McCoy, A. J. et al. Phaser crystallographic software. J Appl Crystallogr 40, 658-674 (2007).

35. Potterton, E., Briggs, P., Turkenburg, M. \& Dodson, E. A graphical user interface to the CCP4 program suite. Acta Crystallogr D Biol Crystallogr 59, 1131-1137 (2003).

36. Shan, S. et al. Crystal structure of 4-diphosphocytidyl-2-C-methyl-D-erythritol kinase (IspE) from Mycobacterium tuberculosis. FASEB J 25, 1577-1584 (2011).

\section{Acknowledgments}

We would like to thank staff members of the Core Facility for Protein Research of the Institute of Biophysics, CAS; especially Yi Han, Ya Wang and Xiaoxia Yu for their technical support. This work was supported by grants from the State Key Development Program for Basic Research of the Ministry of Science and Technology of China (973 Project Grant Nos. 2014CB542800, 2011CB915501 and 2011CB910304), National Science Foundation Grant 813300237, and National Infectious Disease Funding (Grant No. 2012ZX10004701).

\section{Author contributions}

J.L. and X.G. performed experiments, interpreted results and wrote the manuscript. N.S. analyzed data and participated in drafting the article for important intellectual content. W.C., Y.D. and X.X. collected crystallographic data and analyzed it. Z.R. and X.L. initiated the study, co-designed experiments, interpreted data and wrote the manuscript. All authors have contributed to, seen and approved the manuscript.

\section{Additional information}

Accession codes: The co-ordinates and structure factor files for apo- and maltose-bound $\mathrm{MaK}$ have been deposited in the PDB under accession codes $4 \mathrm{O} 7 \mathrm{O}$ and $4 \mathrm{O} 7 \mathrm{P}$, respectively.

Supplementary information accompanies this paper at http://www.nature.com/ scientificreports

Competing financial interests: The authors declare no competing financial interests.

How to cite this article: Li, J. et al. Homotypic dimerization of a maltose kinase for molecular scaffolding. Sci. Rep. 4, 6418; DOI:10.1038/srep06418 (2014).

This work is licensed under a Creative Commons Attribution-NonCommercialNoDerivs 4.0 International License. The images or other third party material in this article are included in the article's Creative Commons license, unless indicated otherwise in the credit line; if the material is not included under the Creative Commons license, users will need to obtain permission from the license holder in order to reproduce the material. To view a copy of this license, visit http:// creativecommons.org/licenses/by-nc-nd/4.0/ 